\title{
Stability Analysis in Couple-Stress Rotatory Fluid
}

\author{
PARDEEP KUMAR \\ Department of Mathematics, ICDEOL, Himachal Pradesh University, \\ Summerhill, Shimla-5, INDIA
}

\begin{abstract}
The aim of the present research was to study the effect of uniform rotation on the layer of a couple-stress fluid heated from below in porous medium. Following the linearized stability theory, Boussinesq approximation and normal mode analysis, the dispersion relation is obtained. The stationary convection, stability of the system and oscillatory modes are discussed. For the case of stationary convection, it is found that rotation has a stabilizing effect, whereas the couple-stress parameter and medium permeability have both stabilizing and destabilizing effects on the system. It is found that the presence of rotation introduces oscillatory modes in the system which were nonexistent in its absence. A sufficient condition for the non-existent of overstability is also obtained.
\end{abstract}

Key-Words: couple-stress fluid, heated from below, linearized stability theory, normal mode analysis method, porous medium, uniform rotation

Received: December 16, 2020. Revised: May 22, 2021. Accepted: June 9, 2021. Published: June 29, 2021.

\section{Introduction}

The thermal instability of a fluid layer heated from below plays an important role in geophysics, oceanography, atmospheric physics etc., and has been investigated by many authors, e.g. Be'nard [1], Rayleigh [2], Jeffreys [3]. A detailed account of the theoretical and experimental results of the onset of thermal instability (Be'nard convection) in a fluid layer under varying assumptions of hydrodynamics and hydromagnetics has been given in a treatise by Chandrasekhar [4] in his celebrated monograph. The use of the Boussinesq approximation has been made throughout, which states that the variations of density in the equations of motion can safely be ignored everywhere except in its association with the external force. The approximation is well justified in the case of incompressible fluids. Abdul-Bari and Al-Rubai [5] have studied the influence of Rayleigh-number in turbulent and laminar region in parallel-plate vertical channels. The influence of radiation on the unsteady free convection flow of a viscous incompressible fluid past a moving vertical plate with Newtonian heating has been investigated theoretically by Narahari and Ishak [6]. Vest and Arpaci [7] have studied the stability of a horizontal layer of Maxwell's viscoelastic fluid heated from below. The nature of instability and some factors may have different effects, on viscoelastic fluids as compared to the Newtonian fluids. For example, Bhatia and Steiner [8] have considered the effect of a uniform rotation on the thermal instability of a Maxwell fluid and have found that rotation has a destabilizing influence, in contrast to the stabilizing effect on a Newtonian fluid. Bhatia and Steiner [9] have also considered the thermal instability of a Maxwell fluid in hydromagnetics and have found that the magnetic field has stabilizing effect on viscoelastic fluid, just as in case of Newtonian fluid. Sharma [10] has studied the thermal instability of a layer of Oldroydian [11] fluid acted on by a uniform rotation and found that rotation has destabilizing and stabilizing effects under certain conditions, in contrast to a Maxwell fluid where the effect is destabilizing.

There are many fluids that cannot be characterized by Maxwell's or Oldroyd's constitutive relations. One type of such fluids are couple-stress fluids. The theory of couplestress fluid has been formulated by Stokes [12]. One of the applications of couple-stress fluid is its use to the study of the mechanisms of lubrications of synovial joints, which has 
become the object of scientific research. A human joint is a dynamically loaded bearing which has articular cartilage as the bearing and synovial fluid as the lubricant. When a fluid film is generated, squeeze-film action is capable of providing considerable protection to the cartilage surface. The shoulder, ankle, knee and hip joints are the loaded-bearing synovial joints of the human body and these joints have a low friction coefficient and negligible wear.

Normal synovial fluid is a viscous, nonNewtonian fluid and is generally clear or yellowish. According to the theory of Stokes [12], couple-stresses appear in noticeable magnitudes in fluids with very large molecules. Many of the flow problems in fluids with couple-stresses, discussed by Stokes, indicate some possible experiments, which could be used for determining the material constants, and the results are found to differ from those of Newtonian fluid. Couple-stresses are found to appear in noticeable magnitudes in polymer solutions for force and couple-stresses. This theory is developed in an effort to examine the simplest generalization of the classical theory, which would allow polar effects. The constitutive equations proposed by Stokes [12] are:

$$
\begin{aligned}
& T_{(i j)}=\left(-p+\lambda D_{k k}\right) \delta_{i j}+2 \mu D_{i j}, \\
& T_{[i j]}=-2 \eta \vec{W}_{i j . k k}-\frac{\rho}{2} \vec{\varepsilon}_{i j s} G_{s},
\end{aligned}
$$

and

$$
M_{i j}=4 \eta \vec{\omega}_{j, i}+4 \eta^{\prime} \vec{\omega}_{i, j}
$$

where

$$
D_{i j}=\frac{1}{2}\left(V_{i, j}+V_{j, i}\right), \vec{W}_{i j}=-\frac{1}{2}\left(V_{i, j}-V_{j, i}\right)
$$

and

$$
\vec{\omega}_{i}=\frac{1}{2} \vec{\varepsilon}_{i j k} V_{k, j}
$$

Here $T_{i j}, T_{(i j)}, T_{[i j]}, M_{i j}, D_{i j}, \vec{W}_{i, j}, \vec{\omega}_{i}, G_{s}, \vec{\varepsilon}_{i j k}$, $\mathrm{V}, \rho$ and $\lambda, \mu, \eta, \eta^{\prime}$, are stress tensor, symmetric part of $T_{i j}$, anti-symmetric part of $T_{i j}$, the couple-stress tensor, deformation tensor, the vorticity tensor, the vorticity vector, body couple, the alternating unit tensor, velocity field, the density and material constants respectively. The dimensions of $\lambda$ and $\mu$ are those of viscosity whereas the dimensions of $\eta$ and $\eta^{\prime}$ are those of momentum.
Since the long chain hyaluronic acid molecules are found as additives in synovial fluids, Walicki and Walicka [13] modeled the synovial fluid as a couple-stress fluid. The synovial fluid is the natural lubricant of joints of the vertebrates. The detailed description of the joint lubrication has very important practical implications. Practically all diseases of joints are caused by or connected with a malfunction of the lubrication. The efficiency of the physiological joint lubrication is caused by several mechanisms. The synovial fluid is, due to its content of the hyaluronic acid, a fluid of high viscosity, near to a gel. Goel et al. [14] have studied the hydromagnetic stability of an unbounded couple-stress binary fluid mixture under rotation with vertical temperature and concentration gradients. Sharma et al. [15] have considered a couple-stress fluid with suspended particles heated from below. They have found that for stationary convection, couple-stress has a stabilizing effect whereas suspended particles have a destabilizing effect. Kumar et al. [16] have considered the thermal instability of a layer of a couple-stress fluid acted on by a uniform rotation, and have found that for stationary convection, the rotation has a stabilizing effect whereas couple-stress has both stabilizing and destabilizing effects. Thermosolutal convection in a couple-stress fluid in presence of magnetic field and rotation, separately, has been investigated by Kumar and Singh [17, 18]. Kumar and Kumar [19] have considered the problem of thermosolutal convection in compressible couple-stress fluid in presence of suspended particles. In another study, Kumar and Kumar [20] have considered the transport of vorticity in couple-stress fluid in the presence of suspended magnetic particles.

In recent years, the investigations of flow of fluids through porous media have become an important topic due to the recovery of crude oil from the pores of reservoir rocks. The study of the onset of convection in a porous medium has attracted considerable interest because of its natural occurrence and of its intrinsic importance in many industrial problems, particularly in petroleum exploration, chemical, and nuclear industries. The derivation of the basic equations of a layer of fluid heated from below in porous medium, using the Boussinesq approximation, has been given by Joseph [21]. The study of a layer of fluid heated from below 
in porous media is motivated both theoretically and by its practical applications in engineering disciplines. Among the applications in engineering disciplines one can find the food process industry, chemical process industry, solidification and centrifugal casting of metals. The development of geothermal power resources has increased general interest in the properties of convection in porous medium. When a fluid permeates an isotropic and homogeneous porous medium, the gross effect is represented by Darcy's law. A great number of applications in geophysics may be found in the books by Phillips [22], Ingham and Pop [23], and Nield and Bejan [24]. Kumar and Kumar [25] have mathematically investigated the triply diffusive convection in couple-stress fluid in the presence of uniform vertical magnetic field through porous medium.

Keeping in mind the importance in geophysics, soil sciences, ground water hydrology, astrophysics, and various applications mentioned above, the present paper is devoted to the linear stability analysis of a layer of couple-stress fluid heated from below in the presence of uniform rotation through porous medium.

\section{Formulation of the Problem and Dispersion Relation}

The present problem is studied using methods of linearized stability theory and normal mode analysis. First of all linearized perturbation equations relevant to the problem are obtained and then dispersion relation obtained by using normal analysis method.

Consider an infinite horizontal incompressible layer of a couple-stress fluid of thickness $d$, heated from below so that the temperatures and densities at the bottom surface $z=0$ are $T_{0}, \rho_{0}$ and at the upper surface $z=d$ are $T_{d}, \rho_{d}$, respectively, and that a uniform adverse temperature gradient $\beta(=|d T / d z|)$ is maintained. This layer is acted on by a uniform rotation $\vec{\Omega}=(0,0, \Omega)$ and the gravity field $\vec{g}=$ $(0,0,-g)$ pervades the system. This fluid layer is flowing through an isotropic and homogeneous porous medium of porosity $\varepsilon$ and the medium permeability $k_{1}$.

Let $\Gamma_{i j}, \tau_{i j}, e_{i j}, \delta_{i j}, \mu, \mu^{\prime}, v_{i}$, and $x_{i}$ denote the stress tensor, shear stress tensor, rate-ofstrain tensor, Kronecker delta, viscosity, couple-stress viscosity, velocity vector and position vector, respectively. The constitutive relations for the couple-stress fluids are

$$
\left.\begin{array}{c}
\Gamma_{i j}=-p \delta_{i j}+\tau_{i j}, \\
\tau_{i j}=2\left(\mu-\mu^{\prime} \nabla^{2}\right) e_{i j}, \\
e_{i j}=\frac{1}{2}\left(\frac{\partial v_{i}}{\partial x_{j}}+\frac{\partial v_{j}}{\partial x_{i}}\right) .
\end{array}\right\}
$$

Let $p, \rho, T$ and $\vec{q}(u, v, w)$ denote the fluid pressure, density, temperature and filter velocity respectively. Then the momentum balance, mass balance and energy balance equations of the couple-stress fluid through porous medium (Stokes [12], Joseph [21]) in the presence of rotation are

$$
\begin{aligned}
& \frac{1}{\varepsilon}\left[\frac{\partial \vec{q}}{\partial t}+\frac{1}{\varepsilon}(\vec{q} \cdot \nabla) \vec{q}\right]=-\nabla\left(\frac{p}{\rho_{0}}-\frac{1}{2}|\vec{\Omega} \times \vec{r}|^{2}\right) \\
& +\vec{g}\left(1+\frac{\delta \rho}{\rho_{0}}\right)-\frac{1}{k_{1}}\left(v-\frac{\mu^{\prime}}{\rho_{0}} \nabla^{2}\right) \vec{q}+\frac{2}{\varepsilon}(\vec{q} \times \vec{\Omega})
\end{aligned}
$$

$\nabla \cdot \vec{q}=0$,

$E \frac{\partial T}{\partial t}+(\vec{q} \cdot \nabla) T=\kappa \nabla^{2} T$.

The equation of state is

$$
\rho=\rho_{0}\left[1-\alpha\left(T-T_{0}\right)\right],
$$

where the suffix zero refers to values at the reference level $z=0$. In writing equation (2), use has been made of the Boussinesq approximation which states that the density variations are ignored in all terms in the equation of motion except the external force term. The kinematic viscosity $v$, thermal diffusivity $\kappa$, couple-stress viscosity $\mu^{\prime}$ and coefficient of thermal expansion $\alpha$ are all assumed to be constants. Here $E=$ $\varepsilon+(1-\varepsilon) \rho_{S} c_{S} /\left(\rho_{0} c_{V}\right)$, is constant where $\rho_{S}, c_{S}$ and $\rho_{0}, c_{V}$ stand for density and heat capacity of solid (porous matrix) material and fluid, respectively and $\vec{r}=(x, y, z)$. 
The basic motionless solution is

$$
\begin{aligned}
& \vec{q}=(0,0,0), T=T_{0}-\beta z, \\
& \rho=\rho_{0}(1+\alpha \beta z) .
\end{aligned}
$$

Assume small perturbations around the basic solution and let $\vec{q}(u, v, w), \theta, \delta \rho$ and $\delta p$ denote, respectively, the perturbations in fluid velocity $(0,0,0)$, temperature $T$, density $\rho$ and pressure $p$. The change in density $\delta \rho$, caused mainly by the perturbation $\theta$ in temperature, is given by

$$
\delta \rho=-\alpha \rho_{0} \theta .
$$

Then the linearized perturbation equations are

$$
\begin{aligned}
\frac{1}{\varepsilon} \frac{\partial \vec{q}}{\partial t}=-\frac{1}{\rho_{0}} \nabla \delta p-\vec{g} & \alpha \theta-\frac{1}{k_{1}}\left(v-\frac{\mu^{\prime}}{\rho_{0}} \nabla^{2}\right) \vec{q} \\
+ & \frac{2}{\varepsilon}(\vec{q} \times \vec{\Omega}),
\end{aligned}
$$

$$
\nabla \cdot \vec{q}=0,
$$

$$
E \frac{\partial \theta}{\partial t}=\beta w+\kappa \nabla^{2} \theta
$$

Writing the scalar components of equation (8) and eliminating $u, v, \delta p$ between them by using (9), we obtain

$$
\begin{aligned}
& \frac{1}{\varepsilon} \frac{\partial}{\partial t} \nabla^{2} w+\frac{1}{k_{1}}\left(v-\frac{\mu^{\prime}}{\rho_{0}} \nabla^{2}\right) \nabla^{2} w \\
& -g \alpha\left(\frac{\partial^{2} \theta}{\partial x^{2}}+\frac{\partial^{2} \theta}{\partial y^{2}}\right)+\frac{2 \Omega}{\varepsilon} \frac{\partial \zeta}{\partial z}=0, \\
& \frac{1}{\varepsilon} \frac{\partial \zeta}{\partial t}+\frac{1}{k_{1}}\left(v-\frac{\mu^{\prime}}{\rho_{0}} \nabla^{2}\right) \zeta=\frac{2 \Omega}{\varepsilon} \frac{\partial w}{\partial z},
\end{aligned}
$$

where

$$
\zeta\left(=\frac{\partial v}{\partial x}-\frac{\partial u}{\partial y}\right)
$$

is the $z$ - component of vorticity.

We now analyze the disturbances into normal modes, assuming that the perturbation quantities are of the form

$$
\begin{gathered}
{[w, \theta, \zeta]=[W(z), \Theta(z), Z(z)] \exp \left(i k_{x} x\right.} \\
\left.+i k_{y} y+n t\right),
\end{gathered}
$$

where $k_{x}, k_{y}$ are wave numbers along the $x$-direction and $y$-direction, respectively, $k\left[=\left(k_{x}^{2}+k_{y}^{2}\right)^{1 / 2}\right]$ is the resultant wave number and $n$ is the growth rate which is, in general, a complex constant.

Using expression (13), equations (10) - (12) in non-dimensional form become

$$
\begin{aligned}
& \quad\left(D^{2}-a^{2}\right)\left[\frac{\sigma}{\varepsilon}+\frac{1}{P_{l}}-\frac{F}{P_{l}}\left(D^{2}-a^{2}\right)\right] W \\
& +\frac{g \alpha d^{2}}{v} a^{2} \Theta+\frac{2 \Omega \mathrm{d}^{3}}{v \varepsilon} D Z=0 \\
& {\left[\frac{\sigma}{\varepsilon}+\frac{1}{P_{l}}-\frac{F}{P_{l}}\left(D^{2}-a^{2}\right)\right] Z-\frac{2 \Omega \mathrm{d}}{v \varepsilon} D W} \\
& =0, \\
& \left(D^{2}-a^{2}-E p_{1} \sigma\right) \Theta=-\left(\frac{\beta d^{2}}{\kappa}\right) W
\end{aligned}
$$

where we have put $a=k d, \sigma=n d^{2} / v, x^{*}=$ $x / d, y^{*}=y / d, z^{*}=z / d$ and $D=d / d z$.

$p_{1}=v / \kappa$ is the Prandtl number, $P_{l}=k_{1} / d^{2}$ is the dimensionless medium permeability and $F=\mu^{\prime} /\left(\rho_{0} d^{2} v\right)$ is the dimensionless couplestress parameter. We shall suppress the stars $(*)$ for convenience hereafter.

Eliminating $\mathrm{Z}$ and $\Theta$ between equations (14) (16), we obtain

$$
\begin{aligned}
& \left(D^{2}-a^{2}\right)\left(D^{2}-a^{2}-E p_{1}\right) \\
& \begin{array}{r}
\left.\frac{\sigma}{\varepsilon}+\frac{1}{P_{l}}-\frac{F}{P_{l}}\left(D^{2}-a^{2}\right)\right]^{2} W \\
\quad+T_{A}\left(D^{2}-a^{2}-E p_{1} \sigma\right) D^{2} W
\end{array} \\
& =R a^{2}\left[\frac{\sigma}{\varepsilon}+\frac{1}{P_{l}}-\frac{F}{P_{l}}\left(D^{2}-a^{2}\right)\right] W
\end{aligned}
$$

where $R=g \alpha \beta d^{4} /(v \kappa)$ is the Rayleigh number and $T_{A}=\frac{4 \Omega^{2} d^{4}}{v^{2} \varepsilon^{2}}$ is the modified Taylor number.

We now assume that the fluid layer is confined between two free boundaries. The case of two free boundaries is of artificial nature, but due to mathematically simplicity it enables us to find analytical solutions and to make some qualitative conclusions. The appropriate 
boundary conditions, with respect to which equations (14) - (16) must be solved, are

$$
\begin{aligned}
& W=D^{2} W=0, \Theta=0, D Z=0 \\
& \text { for } z=0 \text { and } z=1 .
\end{aligned}
$$

Using the boundary conditions (18), it can be shown that all the even order derivatives of $W$ must vanish on the boundaries, and hence the proper solution of (17) characterizing the lowest mode is

$$
W=W_{0} \sin \pi z
$$

where $W_{0}$ is a constant.

Substituting the proper solution (19) in equation (17), we obtain the dispersion relation

$$
\begin{aligned}
& R_{1} \\
& =\frac{\left.\left[(1+x)\left\{\frac{i \sigma_{1}}{\varepsilon}+\frac{1}{P}+\frac{\pi^{2} F}{P}(1+x)\right\}_{1} \sigma_{1}\right)+T_{1}\right]}{x\left(\frac{i \sigma_{1}}{\varepsilon}+\frac{1}{P}+\frac{\pi^{2} F}{P}(1+x)\right)},
\end{aligned}
$$

where

$$
\begin{gathered}
R_{1}=R / \pi^{4}, T_{1}=T_{A} / \pi^{4}, x=a^{2} / \pi^{2}, \\
i \sigma_{1}=\sigma / \pi^{2} \text { and } P=\pi^{2} P_{l} .
\end{gathered}
$$

\section{Important Theorems and Discussion}

Here we discuss the case of stationary convection in Theorem 1, theoretically as well as analytically. In Theorems $2-4$, the case of stability of the system and oscillatory modes discussed. In Theorem 5, the case of overstability discussed in which a sufficient condition for the non-existence of overstability is derived.

Theorem 1: For stationary convection case:

(I) In the absence of rotation, the couple-stress parameter postpones the onset of convection i.e. has a stabilizing effect and in the presence of rotation, the couplestress parameter has both stabilizing and destabilizing effects on the system.

(II) In the absence of rotation, the medium permeability hastens the onset of convection i.e. has a destabilizing effect and in the presence of rotation, the medium permeability has both stabilizing and destabilizing effects on the system.

(III) Rotation postpones the onset of convection i.e. rotation has a stabilizing effect in couple-stress fluid heated from below in porous medium.

Proof: When the instability sets in as stationary convection, the marginal state will be characterized by $\sigma=0$. Putting $\sigma=0$, the dispersion relation (20) reduces to

$R_{1}$

$=\frac{(1+x)\left[(1+x)\left\{1+\pi^{2} F(1+x)\right\}^{2}+P^{2} T_{1}\right]}{x P\left(1+\pi^{2} F(1+x)\right)}$.

which expresses the Rayleigh number $R_{1}$ as a function of the dimensionless wave number $x$ and the parameters $F, P$ and $T_{1}$. To investigate the effects of couple-stress parameter, medium permeability and rotation, we examine the natur es of

analytically.

$$
\frac{d R_{1}}{d F}, \frac{d R_{1}}{d P} \text { and } \frac{d R_{1}}{d T_{1}}
$$

(I) Equation (21) yields

$$
\begin{aligned}
\frac{d R_{1}}{d F}=\frac{\pi^{2}(1+x)^{2}}{x P} & {[(1+x)} \\
- & \left.\frac{P^{2} T_{1}}{\left\{1+\pi^{2} F(1+x)\right\}^{2}}\right] .
\end{aligned}
$$

It is clear from equation (22) that in the absence of rotation, $\frac{d R_{1}}{d F}$ is always positive. So the couple-stress parameter postpones the onset of convection i.e. has a stabilizing effect on the system in the absence of rotation.

In the presence of rotation, if 
$T_{1}<\frac{(1+x)\left\{1+\pi^{2} F(1+x)\right\}^{2}}{P^{2}}$,

the couple-stress parameter postpones the onset of convection, whereas it is clear from equation (22) that if

$T_{1}>\frac{(1+x)\left\{1+\pi^{2} F(1+x)\right\}^{2}}{P^{2}}$.

the couple-stress parameter hastens the onset of convection. Therefore, in the presence of rotation, under condition (23), the couple-stress parameter has stabilizing effect and under condition (24), the couple-stress parameter has a destabilizing effect.

(II) It is evident from equation (21) that

$$
\begin{array}{r}
\frac{d R_{1}}{d P}=\frac{(1+x)}{x\left\{1+\pi^{2} F(1+x)\right\}}\left[T_{1}\right. \\
-\frac{(1+x)}{P^{2}}\{1 \\
\left.\left.+\pi^{2} F(1+x)\right\}^{2}\right] .
\end{array}
$$

It is clear from equation (25) that, for stationary convection, the medium permeability hastens the onset of convection in the absence of rotation i.e. has a destabilizing effect and also hastens the onset of convection in the presence of rotation if (23) holds good, whereas the medium permeability postpones the onset of convection if (24) holds good. The medium permeability thus has both stabilizing and destabilizing effects on the system in the presence of rotation.

(III) It is clear from equation (21) that

$\frac{d R_{1}}{d T_{1}}=\frac{(1+x) P}{x\left\{1+\pi^{2} F(1+x)\right\}}$,

which is always positive. So the rotation postpones the onset of convection i.e. has a stabilizing effect in couple-stress fluid heated from below in porous medium.

Analytical Explanation: The dispersion relation (21) analyzed numerically. In Figure 1a, $R_{1}$ is plotted against $x$ for $F=2,3,4$, and $T_{1}$ $=0$. It is clear that the couple-stress postpones the onset of convection in the absence of rotation as the Rayleigh number increases with the increase in couple-stress parameter. In
Figure $1 b, R_{1}$ is plotted against $x$ for $F=1,2,3$, $4, \mathrm{P}=100$, and $\mathrm{T}_{1}=1$. Here we also find that the couple-stress hastens the onset of convection in the presence of rotation for small wave numbers whereas the couple-stress postpones the onset of convection in the presence of rotation for higher wave numbers. In Figure $2 \mathrm{a}, \mathrm{R}_{1}$ is plotted against $\mathrm{x}$ for $\mathrm{P}=10$, $15,20, F=2$, and $T_{1}=0$. It is clear that the medium permeability hastens the onset of convection in the absence of rotation as the Rayleigh number decreases with the increase in medium permeability parameter. In Figure 2b, $\mathrm{R}_{1}$ is plotted against $\mathrm{x}$ for $\mathrm{P}=10,15,20,25, \mathrm{~F}$ $=2$, and $\mathrm{T}_{1}=100$. Here we also find that the medium permeability hastens the onset of convection in the presence of rotation for small wave numbers whereas the medium permeability postpones the onset of convection in the presence of rotation for higher wave numbers. In Figure $3, \mathrm{R}_{1}$ is plotted against $\mathrm{x}$ for $\mathrm{T}_{1}=100,200,300, \mathrm{P}=2$, and $\mathrm{F}=2$. It is clear that the rotation postpones the onset of convection as the Rayleigh number increases with the increase in rotation parameter.

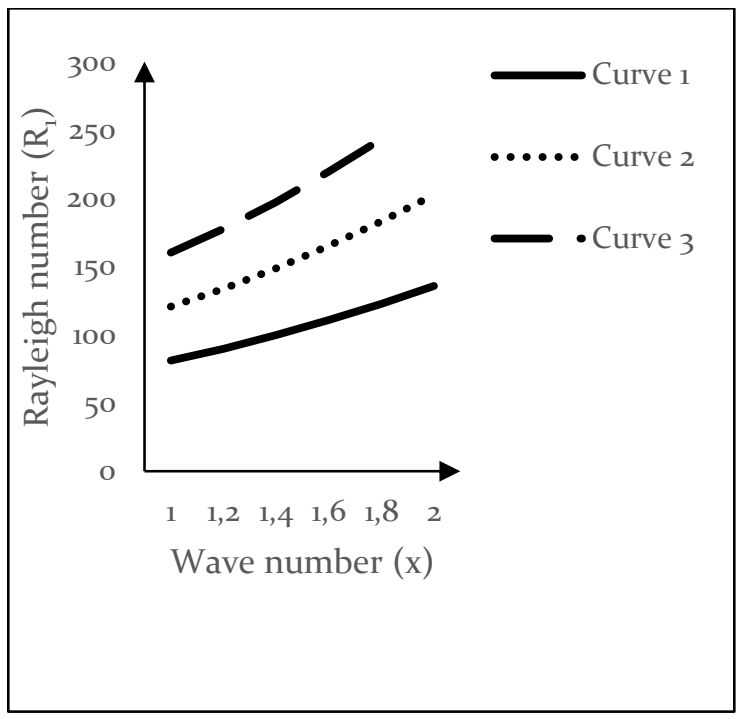

Fig 1a: The variation of Rayleigh number $\left(\mathrm{R}_{1}\right)$ with wave number $(\mathrm{x})$ for $\mathrm{P}=2, \mathrm{~T}_{1}=0, \mathrm{~F}=2$ for Curve $1, F=3$ for Curve 2 and $F=4$ for Curve 3 


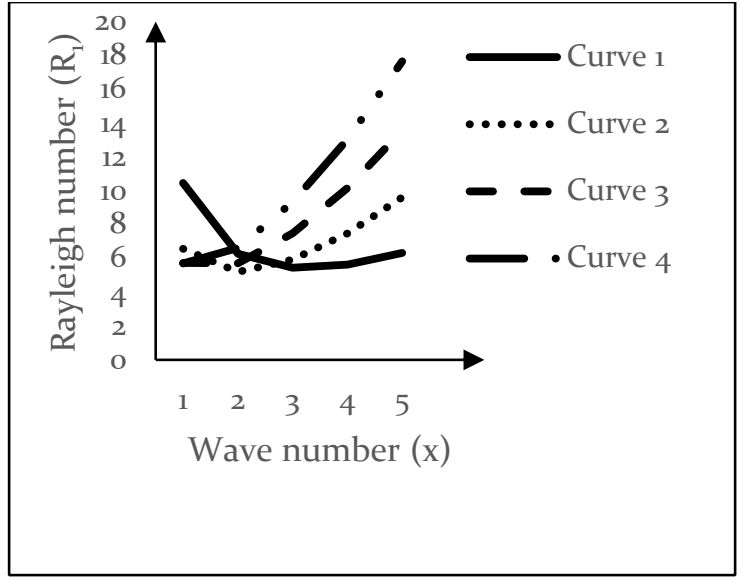

Fig 1b: The variation of Rayleigh number $\left(\mathrm{R}_{1}\right)$ with wave number $(\mathrm{x})$ for $\mathrm{P}=100, \mathrm{~T}_{1}=1, \mathrm{~F}=$ 1 for Curve 1, $F=2$ for Curve 2, $F=3$ for Curve 3 and $\mathrm{F}=4$ for Curve 4

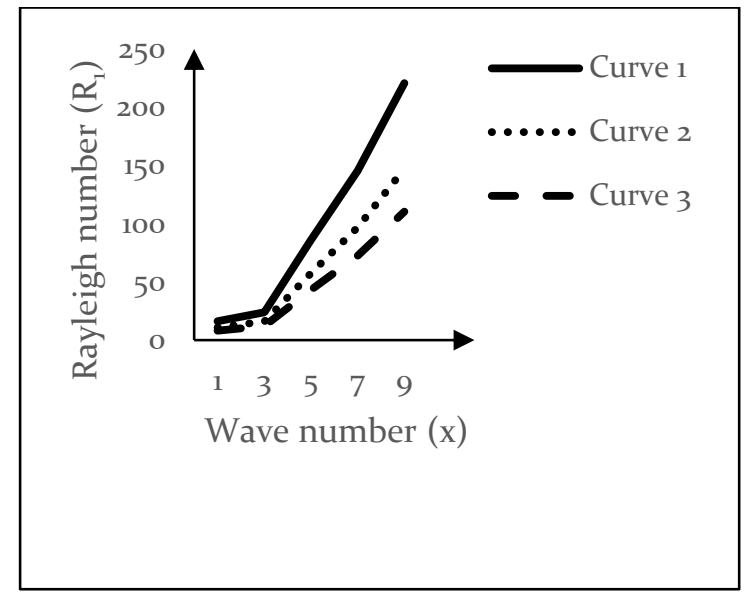

Fig 2a: The variation of Rayleigh number $\left(\mathrm{R}_{1}\right)$ with wave number $(\mathrm{x})$ for $\mathrm{F}=2, \mathrm{~T}_{1}=0, \mathrm{P}=10$ for Curve $1, \mathrm{P}=15$ for Curve 2 and $\mathrm{P}=20$ for Curve 3



Fig 2b: The variation of Rayleigh number $\left(\mathrm{R}_{1}\right)$ with wave number $(\mathrm{x})$ for $\mathrm{F}=2, \mathrm{~T}_{1}=100, \mathrm{P}=$ 10 for Curve $1, \mathrm{P}=15$ for Curve 2, $\mathrm{P}=20$ for Curve 3 and $\mathrm{P}=25$ for Curve 4

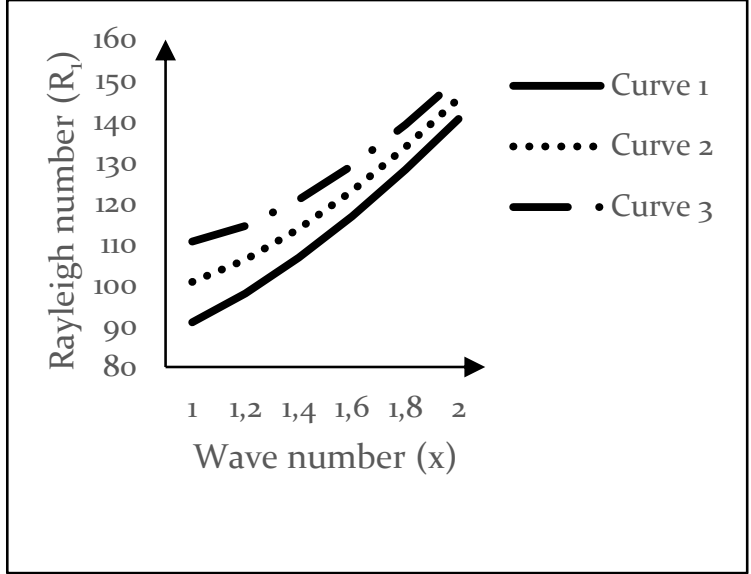

Fig 3: The variation of Rayleigh number $\left(\mathrm{R}_{1}\right)$ with wave number $(\mathrm{x})$ for $\mathrm{F}=2, \mathrm{P}=2, \mathrm{~T}_{1}=$ 100 for Curve $1, T_{1}=200$ for Curve 2 and $T_{1}=$ 300 for Curve 3

Theorem 2: The system is stable or unstable in the presence of couple-stress parameter, rotation and porous medium.

Proof: Multiplying equation (14) by $W^{*}$, the complex conjugate of $W$, integrating over the range of $z$, and making use of equations (15) and (16) together with the boundary conditions (18), we obtain

$$
\begin{aligned}
F I_{1}+\left(1+P_{l} \frac{\sigma}{\varepsilon}\right) & I_{2} \\
& -\frac{g \alpha \kappa a^{2} P_{l}}{v \beta}\left[I_{3}+E p_{1} \sigma^{*} I_{4}\right] \\
& +d^{2}\left[\left(1+P_{l} \frac{\sigma^{*}}{\varepsilon}\right) I_{6}+F I_{5}\right] \\
& =0
\end{aligned}
$$

where

$$
\begin{aligned}
I_{1}=\int_{0}^{1}\left(\left|D^{2} W\right|^{2}\right. & +2 a^{2}|D W|^{2} \\
& \left.+a^{4}|W|^{2}\right) d z, I_{2} \\
& =\int_{0}^{1}\left(|D W|^{2}+a^{2}|W|^{2}\right) d z \\
I_{3}=\int_{0}^{1}\left(|D \Theta|^{2}\right. & \left.+a^{2}|\Theta|^{2}\right) d z, I_{4} \\
& =\int_{0}^{1}|\Theta|^{2} d z, I_{5} \\
& =\int_{0}^{1}\left(|D Z|^{2}+a^{2}|Z|^{2}\right) d z
\end{aligned}
$$


which are all positive definite. Substituting $\sigma=$ $\sigma_{r}+i \sigma_{i}$ and then equating real and imaginary parts of equation (27), we obtain

$$
\begin{aligned}
\sigma_{r}\left[\frac{I_{2}}{\varepsilon}-\frac{g \alpha \kappa a^{2}}{v \beta}\right. & \left.E p_{1} I_{4}+\frac{d^{2}}{\varepsilon} I_{6}\right] \\
& =-\left[\frac{F}{P_{l}} I_{1}+\frac{1}{P_{l}} I_{2}-\frac{g \alpha \kappa a^{2}}{v \beta} I_{3}\right. \\
& \left.+\frac{d^{2}}{P_{l}} I_{6}+\frac{F d^{2}}{P_{l}} I_{5}\right]
\end{aligned}
$$

and

$\sigma_{i}\left[\frac{I_{2}}{\varepsilon}+\frac{g \alpha \kappa a^{2}}{v \beta} E p_{1} I_{4}-\frac{d^{2}}{\varepsilon} I_{6}\right]=0$.

Equation (29) yields that $\sigma_{r}$ may be positive or negative i.e. there may be stability or instability in the presence of couple-stress parameter, rotation and porous medium. The system is, therefore, either stable or unstable.

Theorem 3: The modes may be oscillatory or non-oscillatory in contrast to non-rotatory case.

Proof: Equation (30) yields that $\sigma_{i}=0$ or $\sigma_{i} \neq$ 0 , which means that the modes may be nonoscillatory or oscillatory.

In the absence of rotation, equation (30) reduces to

$\sigma_{i}\left[\frac{I_{2}}{\varepsilon}+\frac{g \alpha \kappa a^{2}}{v \beta} E p_{1} I_{4}\right]=0$,

and the terms in brackets are positive definite. Thus $\sigma_{i}=0$, which means that oscillatory modes are not allowed and the principle of exchange of stabilities is satisfied for the couple-stress fluid heated from below in porous medium in the absence of rotation. It is clear from equation (30) that the presence of rotation brings oscillatory modes (as $\sigma_{i}$ may not be zero) which were non-existent in its absence for a couple-stress fluid layer heated from below in porous medium.

Theorem 4: The system is stable for $\frac{g \alpha \kappa}{v \beta} \leq$ $\frac{27 \pi^{4}}{4}$ and under the condition $\frac{g \alpha \kappa}{v \beta}>\frac{27 \pi^{4}}{4}$, the system becomes unstable.

Proof: From equation (30), it is clear that $\sigma_{i}$ is zero when the quantity multiplying it is not zero and arbitrary when this quantity is zero.
If $\sigma_{i} \neq 0$, then equation (30) gives

$$
\frac{I_{2}}{\varepsilon}=-\frac{g \alpha \kappa a^{2}}{v \beta} E p_{1} I_{4}+\frac{d^{2}}{\varepsilon} I_{6}
$$

Substituting this in equation (29), we get

$$
\begin{aligned}
& \frac{2 \sigma_{r} I_{2}}{\varepsilon}+\frac{F}{P_{l}} I_{1}+\frac{1}{P_{l}} I_{2}+\frac{d^{2}}{P_{l}} I_{6}+\frac{F d^{2}}{P_{l}} I_{5} \\
& =\frac{g \alpha \kappa a^{2}}{v \beta} I_{3},
\end{aligned}
$$

Equation (32) on using Rayleigh-Ritz inequality gives

$$
\begin{aligned}
& \frac{\left(\pi^{2}+a^{2}\right)^{3}}{a^{2}} \int_{0}^{1}|W|^{2} d z \\
& +\frac{\left(\pi^{2}+a^{2}\right)}{a^{2}}\left\{\frac{d^{2}}{P_{l}} I_{6}+\frac{F d^{2}}{P_{l}} I_{5}+\frac{1}{P_{l}} I_{2}+\frac{2 \sigma_{r}}{\varepsilon} I_{2}\right. \\
& \left.+\frac{F}{P_{l}} I_{1}\right\} \leq \frac{g \alpha \kappa}{v \beta} \int_{0}^{1}|W|^{2} d z .
\end{aligned}
$$

Therefore, it follows from equation (33) that

$$
\begin{aligned}
& {\left[\frac{27 \pi^{4}}{4}-\frac{g \alpha \kappa}{v \beta}\right] \int_{0}^{1}|W|^{2} d z} \\
& +\frac{\left(\pi^{2}+a^{2}\right)}{a^{2}}\left\{\frac{d^{2}}{P_{l}} I_{6}+\frac{F d^{2}}{P_{l}} I_{5}+\frac{1}{P_{l}} I_{2}+\frac{2 \sigma_{r}}{\varepsilon} I_{2}\right. \\
& \left.+\frac{F}{P_{l}} I_{1}\right\} \leq 0,
\end{aligned}
$$

since minimum value of $\frac{\left(\pi^{2}+a^{2}\right)^{3}}{a^{2}}$ with respect to $a^{2}$ is $\frac{27 \pi^{4}}{4}$.

Now, let $\sigma_{r} \geq 0$, we necessary have from (34) that

$\frac{g \alpha \kappa}{v \beta}>\frac{27 \pi^{4}}{4}$.

Hence, if

$\frac{g \alpha \kappa}{v \beta} \leq \frac{27 \pi^{4}}{4}$,

then $\sigma_{r}<0$. Therefore, the system is stable. Thus, under condition (36), the system is stable and under condition (35) the system becomes unstable. 
Theorem 5: The sufficient condition for the non-existence of overstability is

$$
\kappa<\left(\frac{v \varepsilon}{k_{1}}\right)^{3}\left(\frac{d^{2}}{4 \Omega^{2} \pi^{2}}\right)\left[\varepsilon+(1-\varepsilon) \frac{\rho_{s} c_{s}}{\rho_{0} c_{v}}\right] .
$$

Proof: Here we discuss the possibility of whether instability may occur as overstability. Since for overstability, we wish to determine the critical Rayleigh number for the onset of instability via a state of pure oscillations, it suffices to find conditions for which equation (20) will admit of solutions with $\sigma_{1}$ real. Equating real and imaginary parts of equation (20), and eliminating $R_{1}$ from the resulting equations, we obtain

$$
\begin{aligned}
& \sigma_{1}^{2}\left[\frac{b}{\varepsilon^{2}}+\frac{E p_{1}}{\varepsilon}\left(\frac{1}{P}+\frac{\pi^{2} F b}{P}\right)\right] b \\
& +\left[b^{2}\left(\frac{1}{P}+\frac{\pi^{2} F b}{P}\right)^{2}+E p_{1} \varepsilon T_{1}\left(\frac{1}{P}+\frac{\pi^{2} F b}{P}\right)\right] \\
& +b\left[E p_{1} \varepsilon\left(\frac{1}{P}+\frac{\pi^{2} F b}{P}\right)^{3}-T_{1}\right] \\
& =0
\end{aligned}
$$

where we have put $b=1+x$.

Since $\sigma_{1}$ is real for overstability, the value of $\sigma_{1}^{2}$ is positive. Equation (37) shows that this is impossible if

$T_{1}<\frac{E p_{1} \varepsilon}{P^{3}}$,

which means that

$$
\kappa<\left(\frac{v \varepsilon}{k_{1}}\right)^{3}\left(\frac{d^{2}}{4 \Omega^{2} \pi^{2}}\right)\left[\varepsilon+(1-\varepsilon) \frac{\rho_{s} c_{s}}{\rho_{0} c_{v}}\right] .
$$

The condition (39) is, therefore, a sufficient condition for the non-existence of overstability, the violation of which does not necessarily imply the occurrence of overstability.

\section{Conclusions}

With the growing importance of nonNewtonian fluids in modern technology and industries, investigation of such fluids is desirable. Stokes [12] formulated the theory of couple-stress fluid. One of the applications of couple-stress fluid is its use in the study of mechanisms of lubrications of synovial joints, which has become the object of scientific research. A human joint is a dynamically loaded bearing that has articular cartilage as the bearing and synovial fluid as the lubricant. The shoulder, ankle, knee, and hip joints are the loaded-bearing synovial joints of the human body. Since long chain hyaluronic acid molecules are found as additives in synovial fluids, Walicki and Walicka modeled the synovial fluid as a couple-stress fluid. Therefore, an attempt has been made to investigate the effect of uniform rotation on a layer of couple-stress fluid heated from below in porous medium. The main conclusions from the analysis of this paper are as follows:

- For the case of stationary convection, the couple-stress parameter and medium permeability has both stabilizing and destabilizing effects on the system whereas the rotation has a stabilizing effect on the system.

- It is found that rotation introduces oscillatory modes in the system which was non-existent in its absence.

- It is observed that the system is stable for $\frac{g \alpha \kappa}{v \beta} \leq \frac{27 \pi^{4}}{4}$ and under the condition $\frac{g \alpha \kappa}{v \beta}>\frac{27 \pi^{4}}{4}$, the system becomes unstable.

- The case of overstability is also considered. The condition

$$
\begin{aligned}
& \kappa<\left(\frac{v \varepsilon}{k_{1}}\right)^{3}\left(\frac{d^{2}}{4 \Omega^{2} \pi^{2}}\right) {[\varepsilon} \\
&+(1-\varepsilon) \frac{\rho_{s} c_{s}}{\rho_{0} c_{v}}
\end{aligned}
$$

is the sufficient condition for the nonexistence of overstability, the violation of which does not necessarily imply the occurrence of overstability.

\section{Acknowledgements}

The author is grateful to all the three learned referees for their useful technical comments and valuable suggestions, which led to a significant improvement of the paper. 


\section{References}

[1] Be'nard, H., Les Tourbillions Cellularies Dans Une Nappe Liquide, Revue Gene'rale des Sciences Pures et Applique'es, Vol. 11, 1900, pp. 1261-1271, 1309-1328.

[2] Rayleigh, L., On convective currents in a horizontal layer of fluid when the higher temperature is on the underside, Phil. Mag., Vol. 32, 1916, pp. 529-546.

[3] Jeffreys, H., The stability of a fluid layer heated from below, Phil. Mag., Vol. 2, 1926, pp. 833-844.

[4] Chandrasekhar, S., Hydrodynamic and Hydromagnetic Stability, Dover Publication, New York 1981.

[5] Abdul-Bari, H.A. and Al-Rubai, R.T.A., Influence of number in turbulent and laminar region in parallel-plate vertical channels, Asian J. Applied Sci., Vol. 1, 2008, pp. 265-285.

[6] Narahari, M. and Ishak, A., Radiation effects on free convection flow near a moving vertical plate with Newtonian heating, J. Applied Sci., Vol. 11, 2011, pp. 1096-1104.

[7] Vest, C.M. and Arpaci, V.S., Overstability of a viscoelastic fluid layer heated from below, J. Fluid Mech., Vol. 36, 1969, pp. 613-619.

[8] Bhatia, P.K. and Steiner, J.M., Convective instability in a rotating viscoelastic fluid layer, $Z$. Angew. Math. Mech., Vol. 52, 1972, pp. 321-327.

[9] Bhatia, P.K. and Steiner, J.M., Thermal instability in a viscoelastic fluid layer in hydromagnetics, J. Math. Anal. Appl., Vol. 41, 1973, pp. 271-283.

[10] Sharma, R.C., Effect of rotation on thermal instability of a viscoelastic fluid, Acta Phys. Hung., Vol. 40, 1976, pp. 11-17.

[11] Oldroyd, J.G., Non-Newtonian effects in steady motion of some idealized elastico-viscous liquid, Proc. Roy. Soc. London, Vol. A245, 1958, pp. 278297.

[12] Stokes, V.K., Couple-stresses in fluids, Phys. Fluids, Vol. 9, 1966, pp. 1709-1715.

[13] Walicki, E. and Walicka, A., Inertia effect in the squeeze film of a couple-stress fluid in biological bearing, Appl. Mech. Engng., Vol. 4, 1999, pp. 363-373.
[14] Goel., A.K., Agrawal, S.C. and Agrawal, G.S., Hydromagnetic stability of an unbounded couple stress binary fluid mixture having vertical temperature and concentration gradients with rotation, Ind. J. Pure Appl. Maths., Vol. 30, 1999, pp. 991-1001.

[15] Sharma, R.C., Sunil, Sharma, Y.D. and Chandel, R.S., On couple-stress fluid permeated with suspended particles heated from below, Arch. Mech., Vol. 54, 2002, pp. 287-298.

[16] Kumar, P., Lal, R. and Sharma, P., Effect of rotation on thermal instability in couple-stress elastico-viscous fluid, Z. Fur. Naturforschung A, Vol. 59, 2004, pp. 407-411.

[17] Kumar, P. and Singh, M., Magnetothermosolutal convection in a couple-stress fluid, Ganita Sandesh, India, Vol. 22, 2008, pp. 147-160.

[18] Kumar, P. and Singh, M., Rotatory thermosolutal convection in a couple-stress fluid, Zeitschrift fur Naturforschung, Vol. 64a, 2009, pp. 448-454.

[19] Kumar, P. and Kumar, V., Convection in compressible couple-stress dusty fluid, $J$. Experimental \& Appl. Mech., Vol. 5(2), 2014, pp. 27-37.

[20] Kumar, P. and Kumar, V., Analysis of vorticity transport in magnetic couple-stress fluid, WSEAS Transactions on Applied and Theoretical Mechanics, Vol. 12, 2017, pp. 157-162.

[21] Joseph, D.D., Stability of Fluid Motions, Springer-Verlag Berlin, Vol. I and II., 1976.

[22] Phillips, O.M., Flow and Reaction in Permeable Rocks, Cambridge University Press, Cambridge, UK, 1991.

[23] Ingham, D.B. and Pop, I., Transport Phenomena in Porous Medium, Pergamon Press, Oxford, UK, 1998.

[24] Nield, D.A. and Bejan, A., Convection in porous medium ( $2^{\text {nd }}$ edition), Springer New York, 1999.

[25] Kumar, V. and Kumar, P., On hydromagnetic triply diffusive convection in couple-stress fluid through porous medium, Int. J. Engng. Res. \& Management Tech., Vol. 4(4), 2017, pp. 100-112.

\section{Creative Commons Attribution License 4.0 (Attribution 4.0 International, CC BY 4.0)}

This article is published under the terms of the Creative Commons Attribution License 4.0

https://creativecommons.org/licenses/by/4.0/deed.en_US 